\title{
High-performance electroluminescent refrigeration enabled by photon tunneling
}

\author{
Xianglei Liu ${ }^{1,2}$, Zhuomin M. Zhang ${ }^{1, \text { a) }}$ \\ ${ }^{1}$ G.W. Woodruff School of Mechanical Engineering, \\ Georgia Institute of Technology, Atlanta, Georgia 30332, USA \\ ${ }^{2}$ School of Energy and Power Engineering, Nanjing University of Aeronautics \\ and Astronautics, Nanjing 210016, China
}

\begin{abstract}
Electroluminescent refrigeration, though theoretically proposed half a century ago, is rarely reported due to the requirement of extremely low nonidealities. Here, we theoretically show that by operating the device in the near-field regime with a vacuum gap down to $10 \mathrm{~nm}$, photon tunneling through evanescent waves can increase the tolerance of non-intrinsic nonradiative recombination to 31.6\%. More importantly, the refrigeration rate may be enhanced by 2000 -fold over the far-field scenario. In addition, the lowest achievable cooling temperature against the ambient condition of $300 \mathrm{~K}$ extends from $284.2 \mathrm{~K}$ to $270.6 \mathrm{~K}$. A self-consisted model based on the fluctuationdissipation theory combined with dyadic Green's function method is developed considering the effect of the chemical potential of photons on the energy of Planck's quantum oscillators. This work opens a route to greatly enhance electroluminescent refrigeration, while relieving the strict material's requirement, for solid-state noncontact thermal management.
\end{abstract}

Keywords: Electroluminescent refrigeration, evanescent waves, near-field radiation, photon chemical potential, photon tunneling

\footnotetext{
a) Corresponding author: Tel: (404) 385-4225, Fax: (404) 894-8496, E-mail: zhuomin.zhang@me.gatech.edu
} 


\section{Graphical Abstract}
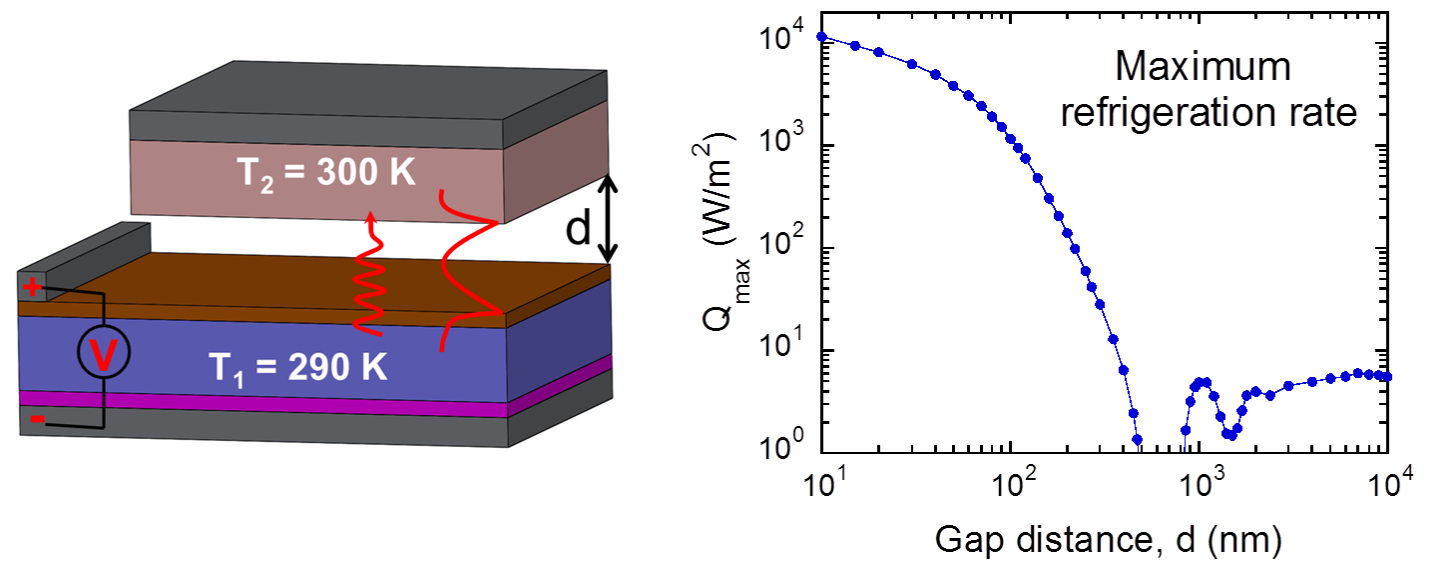


\section{Introduction}

When applying a forward bias to a $p$ - $n$ diode, electrons and holes will be driven into the junction area with at least some of them recombining radiatively. If the energy carried by these outgoing photons is higher than the input electric power, a cooling process called electroluminescence refrigeration occurs through pumping heat from the lattice $[1,2]$. This refrigeration technique has several unique advantages over other solidstate cooling approaches like conventional thermoelectric refrigerators, such as higher efficiencies, easier integration with other optoelectronic devices, and broader operation temperature. In the past few decades, there have been some models proposed to simulate and predict the potential of biased semiconductors in electroluminescence refrigeration [3-10]. Dousmanis et al. predicted that the electroluminescent cooling is achievable if the quantum efficiency of forward-biased GaAs didoes is higher than 97\% [2]. Berdahal [4] discussed the refrigeration effect from a biased junction to an external object of different temperatures considering only radiative recombination and assuming the extraction efficiency to be unity. Later, several groups made some improvements by including nonradiative recombination processes [3,5-11]. However, few of them consider the contributions of low-frequency phonon modes or intraband transitions, which inexorably always lead to a flux flowing from the hot side to the cold side and thus offset the refrigeration effect. Very recently, based on the condition of ultralow applied bias, the electroluminescence refrigeration effect was experimentally observed [12]. However, the obtained refrigeration rate is extremely small, about three orders of magnitude lower than the thermal emission from the environment. Therefore, achieving effective electroluminescent refrigeration, especially when considering various nonidealities 
including the nonradiative recombination and subbandgap radiative energy exchange, remains to be a daunting challenge.

The emission from a biased semiconductor via radiative recombination consists of both propagating waves and evanescent waves. When the biased junction and the ambient object are far apart, as assumed by most previous works, only propagating waves contribute to the energy exchange while the evanescent waves die down before reaching the ambient object due to the inherent exponential decay. It becomes a different story when they are placed in a close proximity. Recent experimental and theoretical works have demonstrated that when the gap distance between two objects of different temperatures is smaller than the characteristic wavelength (around $10 \mu \mathrm{m}$ at room temperature), radiative energy exchange rate can be orders of magnitude greater than the blackbody limit due to photon tunneling that can be explained in wave theory as the coupling of evanescent waves [13-22]. Although the radiation emitted from biased semiconductors is different from conventional Planck's thermal radiation due to the nonzero chemical potential of photons, similar near-field phenomenon involving photon tunneling may occur when the biased low-temperature junction is in a close proximity with a substrate at ambient temperature. Then, evanescent waves may play an important role in mediating radiative energy exchange and thus boosting both the efficiency and refrigerate rate of the biased junction. Chen et al. [23] investigated the energy transfer from a forward-biased junction to a higher temperature object considering contributions of evanescent waves and phonon modes. They predicted that refrigeration effects against a temperature difference of $10 \mathrm{~K}$ could exist only when the gap spacing ranges from tens to hundreds of nanometers by treating the diode as a homogeneous material whose 
chemical potential is increased via voltage biasing. In the present work, we develop a model that can take into account the position dependence of the chemical potential of photons in the diode based on fluctuation-dissipation theory combined with dyadic Green's function approach for a multilayered system. By employing dedicated choices of materials, we show that both near- and far-field refrigeration can be achieved for certain range of temperature differences and the refrigeration rate can be improved by orders of magnitude by operating at the nanoscale.

\section{Theory and methods}

The considered configuration is schematically shown in Fig. 1a. The bottom object (emitter) is a standard GaSb p-i-n junction sandwiched by a top Ag strip and bottom $\mathrm{Ag}$ substrate acting as the anode and cathode, respectively. The top object (receiver) is a Ge film with a thickness of $h_{\mathrm{Ge}}$ on the Ag substrate. Unless specified, the thickness of $p$-typed doped GaSb $h_{\mathrm{p}}=0.1 \mu \mathrm{m}, n$-type doped GaSb $h_{\mathrm{n}}=0.1 \mu \mathrm{m}$, intrinsic GaSb $h_{\mathrm{i}}=1 \mu \mathrm{m}$, and $h_{\mathrm{Ge}}=500 \mu \mathrm{m}$. The bottom and top objects are separated by a gap distance of $d$ and are maintained at a lower temperature of $T_{1}$ and higher temperature $T_{2}=$ $300 \mathrm{~K}$, respectively. The optical constants of Ge are taken from the tabulated values in Ref. [24]. The dielectric function of $\mathrm{Ag}$ in terms of the angular frequency $\omega$ is calculated using a simple Drude model: $\varepsilon_{\mathrm{Ag}}(\omega)=1-\omega_{\mathrm{p}}^{2} /\left(\omega^{2}+i \gamma \omega\right)$ with a scattering rate $\gamma=2.73 \times 10^{13} \mathrm{rad} / \mathrm{s}$ and plasma frequency $\omega_{\mathrm{p}}=1.37 \times 10^{16} \mathrm{rad} / \mathrm{s}$ [14]. The optical constants of GaSb is obtained from Ref. [24] for the ultraviolet and visible regions, from Ferrini et al. [25] for the near-infrared region, and from Ref. [24] and Ref. [26] for the 
mid-infrared region, respectively. At longer wavelengths beyond $30 \mu \mathrm{m}$, a Lorentz model is used to predict the dielectric function $\varepsilon(\omega)=\varepsilon_{\infty}\left(\omega^{2}-\omega_{\mathrm{LO}}^{2}+j \gamma \omega\right) /\left(\omega^{2}-\omega_{\mathrm{TO}}^{2}+j \gamma \omega\right)$, where $\quad \varepsilon_{\infty}=14.63, \quad \gamma=3.35 \times 10^{11} \mathrm{rad} / \mathrm{s}, \quad \omega_{\mathrm{TO}}=4.34 \times 10^{13} \mathrm{rad} / \mathrm{s}, \quad$ and $\omega_{\mathrm{LO}}=4.53 \times 10^{13} \mathrm{rad} / \mathrm{s}$ for GaSb [27]. For doped GaSb, an extra Drude term, $-15.1 \omega_{\mathrm{p}}^{2} /\left(\omega^{2}+j \gamma_{\mathrm{D}} \omega\right)$, is added to the dielectric function to account for contributions of free electrons induced by doping. In this work, $\omega_{\mathrm{p}}=2.60 \times 10^{13} \mathrm{rad} / \mathrm{s}$ and scattering rate $\gamma_{\mathrm{D}}=2.15 \times 10^{13} \mathrm{rad} / \mathrm{s}$ are taken based on a $\mathrm{Zn}$-doped $p$-type GaSb at the doping level of $1.3 \times 10^{17} \mathrm{~cm}^{-3}$ [28]. Note that $n$-type GaSb may have different values of plasma frequency and scattering rate since the effective mass of holes and electrons may be different or the two doping levels are different. Increasing and decreasing $\omega_{\mathrm{p}}$ by $50 \%$ for the $n$-doped and $p$-doped GaSb, respectively or vice versa only reduce the maximum refrigeration rate by less than $3 \%$ for the gap distance of $10 \mu \mathrm{m}$ and becomes trivial for nanoscale gap distance. Therefore, in order to make the analysis easier without losing generalities, we assume the $n$-type and $p$-type GaSb have the same doping concentration and dielectric function.

The working principle of electroluminescent refrigeration is schematically shown in Fig. 1b. An external voltage $V$ is applied through the electrodes on the emitter. For simplicity, it is assumed the voltage only appears across the active intrinsic GaSb layer, so that the difference of Fermi level between $n$-doped and $p$-doped region is $q V$, where $q$ is the elementary charge. Electrons (filled circles) and holes (unfilled circles) are driven into the active region, where photons above the bandgap achieve equilibrium with the 
biased conduction and valence band through radiative generation and recombination. As a result, the photons emitted, including propagating waves, evanescent waves, and ultrahigh wavevector $(k)$ modes, have a nonzero chemical potential of $q V$ [29]. Besides radiative recombination, electrons and holes may also recombine nonradiatively, by which the energy is lost to phonons instead, as shown by the dash line with arrow in Fig. 1b. When the energy carried by the emitted above-bandgap photons is greater than the sum of the electric energy input and the energy flowing reversely due to below-bandgap photons, the refrigeration effect will occur. When the biased junction and the ambient object is far away, only propagating waves contribute to the energy exchange. At submicron the gap distance, some evanescent waves are able to tunnel through, leading to the enhancement of electroluminescent refrigeration rate, but the ultrahigh $k$ modes die down before arriving at the other object. Further reducing the gap distance to the nanoscale can enable photon tunneling for even higher $k$ modes, resulting in an increased refrigeration rate.

Photons outside the active region do not interact with the biased electron-hole system and photons at frequencies below the energy bandgap do not have enough energy to excite the interband transitions. Therefore, these photons are in separate equilibrium with the lattice system, leading to a conventional Planck distribution with a vanishing chemical potential. Subsequently, the mean energy of Planck's oscillator that depends on the position and applied voltage can be expressed as follows:

$$
\Theta(\omega, z, T, V)=\left\{\begin{array}{c}
\frac{\hbar \omega}{\mathrm{e}^{(\hbar \omega-q V) / k_{\mathrm{B}} T}-1}, \omega>\omega_{\mathrm{g}} \text { and } h_{\mathrm{n}}<z<h_{\mathrm{n}}+h_{\mathrm{i}} \\
\frac{\hbar \omega}{\mathrm{e}^{\hbar \omega / k_{\mathrm{B}} T}-1}, \text { otherwise }
\end{array}\right.
$$


where $\hbar$ is the reduced Planck constant, $k_{\mathrm{B}}$ is the Boltzmann constant, and $\omega_{\mathrm{g}}=$ $1.103 \times 10^{15} \mathrm{rad} / \mathrm{s}$ that corresponds to the bandgap energy $\left(E_{\mathrm{g}}=0.726 \mathrm{eV}\right)$ of GaSb.

Since there is a nonuniform distribution of the chemical potential of photons due to the nonequilibrium condition induced by the applied voltage, Kirchhoff's law cannot be directly applied even for the far-field radiative properties of a biased junction. Chen et al. [23] considered whole diode as the active layer by neglecting heavily doped regimes and treating the electrodes as perfect conductors. Here, we employ the fluctuationdissipation theory together with the dyadic Green's function to calculate the radiative energy exchange of the multilayer configuration shown in Fig. 1. Random currents caused by thermal motion of the charge carriers are attributed to the emitted electromagnetic waves. The correlation of these random currents is given by the fluctuation-dissipation theory as $[13,30]$

$$
\left\langle J_{i}(\mathbf{r}, \omega) J_{k}^{*}\left(\mathbf{r}^{\prime}, \omega^{\prime}\right)\right\rangle=\frac{4 \omega \varepsilon_{0} \Theta(\omega, z, T, V) \varepsilon_{i k}^{\prime \prime}(\mathbf{r}, \omega)}{\pi} \delta\left(\mathbf{r}-\mathbf{r}^{\prime}\right) \delta\left(\omega-\omega^{\prime}\right)
$$

where subscripts $i$ or $k$ refer to the vector component, $\varepsilon_{0}$ is the vacuum permittivity, $\varepsilon_{i k}^{\prime \prime}$ is the imaginary part of the dielectric tensor component, and $\delta\left(\mathbf{r}-\mathbf{r}^{\prime}\right)$ or $\delta\left(\omega-\omega^{\prime}\right)$ is the Dirac delta function indicating spatial or temporal incoherence, respectively. For isotropic media, $\varepsilon_{i k}^{\prime \prime}=\varepsilon^{\prime \prime} \delta_{i k}$ where $\varepsilon^{\prime \prime}$ is the imaginary part of the dielectric function and $\delta_{i k}$ is the Kronecker delta $[13,14]$. Note that Eq. (2) is applicable to both high and low frequency photons inside or outside the active regime, provided $\Theta(\omega, z, T, V)$ is modified accordingly. 
The electric field and magnetic field at any position $\mathbf{r}$ are related to the source current at $\mathbf{r}^{\prime}$ by the corresponding electric and magnetic dyadic Green's function $\overline{\bar{G}}^{E}\left(\mathbf{r}, \mathbf{r}^{\prime}, \omega\right)$ and $\overline{\bar{G}}^{H}\left(\mathbf{r}, \mathbf{r}^{\prime}, \omega\right)[14]$

$$
\begin{aligned}
& \mathbf{E}(\mathbf{r}, \omega)=j \omega \mu_{0} \int_{V^{\prime}} \overline{\bar{G}}^{E}\left(\mathbf{r}, \mathbf{r}^{\prime}, \omega\right) \cdot \mathbf{J}\left(\mathbf{r}^{\prime}, \omega\right) d \mathbf{r}^{\prime} \\
& \mathbf{H}(\mathbf{r}, \omega)=\int_{V^{\prime}} \overline{\bar{G}}^{H}\left(\mathbf{r}, \mathbf{r}^{\prime}, \omega\right) \cdot \mathbf{J}\left(\mathbf{r}^{\prime}, \omega\right) d \mathbf{r}^{\prime}
\end{aligned}
$$

where $j=\sqrt{-1}$ and $\mu_{0}$ is the vacuum permeability. The radiative energy flux from the biased emitter to the receiver $Q_{1 \rightarrow 2}$ can be obtained as

$$
Q_{1 \rightarrow 2}=\int_{0}^{\infty} \frac{1}{2}\left\langle\operatorname{Re}\left[\mathbf{E}(\mathbf{r}, \omega) \times \mathbf{H}^{*}(\mathbf{r}, \omega)\right]\right\rangle d \omega
$$

Here, the integrand is the Poynting vector or spectral energy flux $Q_{\omega, 1 \rightarrow 2}$ given by

$$
Q_{\omega, 1 \rightarrow 2}(\omega)=\frac{2 k_{0}^{2}}{\pi} \Theta\left(\omega, z, T_{1}, V\right) \int_{-\infty}^{z_{1}} \operatorname{Re}\left[j \sum_{i=x, y, z} \varepsilon_{i i}^{\prime \prime}(\omega, z)\left(G_{x i}^{E} G_{y i}^{H^{*}}-G_{y i}^{E} G_{x i}^{H^{*}}\right)\right] d z
$$

where $z_{1}=h_{\mathrm{n}}+h_{\mathrm{i}}+h_{\mathrm{p}}$ and $k_{0}$ is the wavevector in vacuum. The radiative energy flux from the receiver to the emitter $Q_{2 \rightarrow 1}$ can be obtained similarly, and the net radiative heat transfer from 1 to 2 is $Q_{12}=Q_{1 \rightarrow 2}-Q_{2 \rightarrow 1}$. The position and frequency dependent $\overline{\bar{G}}^{E}\left(\mathbf{r}, \mathbf{r}^{\prime}, \omega\right)$ and $\overline{\bar{G}}^{H}\left(\mathbf{r}, \mathbf{r}^{\prime}, \omega\right)$ for this multilayer arrangement are obtained analytically by employing the transfer matrix method [31-33]. Note that the Ag coatings are assumed to be sufficiently thick for the top and bottom Ag layer to be treated as semi-infinite. The algorithm developed here allows full calculation of the radiative energy exchange (or heat transfer) between the hot and cold regions, even though certain layers have nonzero chemical potential due to voltage biasing. 
Besides the energy exchange to the receiver, there is an electric power input to the emitter, which can be expressed as $I_{\text {tot }} V$, where $I_{\text {tot }}$ is the total applied current (per unit area). Assuming there is no dissipation caused by recombination outside the active layer, $I_{\text {tot }}=I_{\text {rad }}+I_{\text {Auger }}$, where $I_{\text {rad }}$ is the contribution from radiative recombination and $I_{\text {Auger }}$ comes from the Auger recombination, which is an intrinsic nonradiative recombination. For the moment, let us assume that the semiconductor materials are of very high quality, such that only intrinsic Auger effect contributes to the nonradiative recombination. Employing the reciprocity principle of Green's function, $I_{\text {rad }}$ can be expressed as

$$
I_{\mathrm{rad}}=\frac{2 q}{\pi} \int_{\omega_{g}}^{\infty} k_{0}^{2}\left[\frac{1}{\mathrm{e}^{(\hbar \omega-q V) / k_{\mathrm{B}} T_{1}}-1}-\frac{1}{\mathrm{e}^{\hbar \omega / k_{\mathrm{B}} T_{2}}-1}\right] F(\omega) d \omega
$$

where

$$
F(\omega)=\int_{h_{n}}^{h_{n}+h_{i}} \operatorname{Re}\left[j \sum_{i=x, y, z} \varepsilon_{i i}^{\prime \prime}(\omega, z)\left(G_{x i}^{E} G_{y i}^{H^{*}}-G_{y i}^{E} G_{x i}^{H^{*}}\right)\right] d z
$$

While Eqs. (4) and (5) are for the energy exchange, Eq. (6) calculates the number flux of only above-bandgap photons multiplied by the elementary charge. Although in Eq. (5) the integration extends to the whole $p-i-n$ region, the integration in Eq. (6) is over the active region because only the radiative recombinations and regenerations occurring in the $i$-region contribute to the photocurrent. The first term in the bracket of Eq. (6) represents the current consumption due to radiative recombination. The second term is related to the current produced due to radiative regeneration by absorbing photons emitted by the ambient object. Furthermore, $I_{\text {Auger }}$ can be expressed as 


$$
I_{\text {Auger }}=q h_{\mathrm{i}}\left(C_{n} n+C_{p} p\right)\left(n p-n_{\mathrm{i}}^{2}\right)
$$

where $n$ and $p$ are the electron and hole concentrations, respectively, which are assumed the same in this work as mentioned previously, the sum of the Auger coefficients $C_{n}+C_{p}$ is taken as $5 \times 10^{-30} \mathrm{~cm}^{6} \mathrm{~s}^{-1}$ [27], and the intrinsic carrier density $n_{\mathrm{i}}=$ $1.5 \times 10^{12} \mathrm{~cm}^{-3}[34]$. Then, the rate of heat removal from the emitter (per unit area), called the refrigeration rate or net cooling rate, can be expressed as

$$
Q_{\text {out }}=Q_{12}-I_{\text {tot }} V
$$

The coefficient of performance ( $\beta_{\mathrm{COP}}$ ) of the refrigeration system is defined as

$$
\beta_{\mathrm{COP}}=\frac{Q_{\text {out }}}{I_{\text {tot }} V}
$$

\section{Results and discussion}

The refraction index and extinction coefficient of intrinsic and doped GaSb as well as Ge are shown in Fig. 2. It can be seen that the Drude term modifies the optical response of $\mathrm{GaSb}$ only for the low-frequency region below $3 \times 10^{14} \mathrm{rad} / \mathrm{s}$, as expected. Below $\omega_{\mathrm{g}}$, the net spectral energy exchange between the emitter and receiver is negative since $T_{1}<T_{2}$. However for high frequencies above the bandgap energy, the net radiative energy can flow in an opposite direction from the cold emitter to the hot receiver when $\Theta\left(\omega, z, T_{1}, V\right)$ is greater than $\Theta\left(\omega, z, T_{2}, 0\right)$, i.e., $V>\left(1-T_{1} / T_{2}\right) \hbar \omega / q$. Fig. 3a shows the refrigeration rate from the emitter $Q_{\text {out }}$ as a function of the applied voltage $V$ for $T_{1}=$ $290 \mathrm{~K}$ and $d=10 \mu \mathrm{m}$, which lies in the far-field regime and further increasing $d$ can hardly affect $Q_{\text {out }}$. As expected, in order to achieve refrigeration effects (positive $Q_{\text {out }}$ ), 
the applied voltage $V$ should be high enough so that the positive spectral energy exchange above the bandgap can surpass the low-frequency negative values and the input electric power. If $V$ is very high but still below the threshold $\hbar \omega_{g} / q$, it can be shown that $I_{\text {tot }} V$ scales with $V \exp \left(3 q V / 2 k_{B} T_{1}\right)$ while $Q_{12}$ scales with $\exp \left(q V / k_{\mathrm{B}} T_{1}\right)$, leading to a negative $Q_{\text {out }}$ at a sufficiently high $V$. Therefore, there exists an optimal applied voltage that gives maximum refrigeration rate. For $d=10 \mu \mathrm{m}$, the maximum refrigeration rate (denoted by $Q_{\max }$ here after) against a temperature difference of $10 \mathrm{~K}$ is $5.96 \mathrm{~W} / \mathrm{m}^{2}$ at $V=0.49 \mathrm{~V}$, as can be seen from Fig. 3a.

Pushing the two objects into the close proximity with $d=100 \mathrm{~nm}$ and $10 \mathrm{~nm}$, as shown in Fig. $3 \mathrm{~b}$ and 3c, $Q_{\text {out }}$ versus $V$ has a similar trend but $Q_{\max }$ is increased by more than two orders of magnitude to $1175 \mathrm{~W} / \mathrm{m}^{2}$ when $d=100 \mathrm{~nm}$ and is further enhanced by ten-fold when $d=10 \mathrm{~nm}$. This is because for large gap separations, only propagating waves contribute to the energy exchange, while for small $d$, evanescent waves start to tunnel through and play a dominant role especially at nanometer gap distances. Similar photon tunneling effects of Planck's thermal radiation have been demonstrated to lead to highly efficient radiative energy transport [35-38]. Compared with the performance from Chen et al. [23], the maximum refrigeration rate here is more than two orders of magnitude larger and the refrigeration effect against a temperature difference of $10 \mathrm{~K}$ is extended even to the far field. The main mechanisms for this improvement can be attributed to the low Auger recombination rate of GaSb, a good match of optical response of GaSb and Ge above their similar bandgaps, as shown in Fig. 2b, and the lack of 
phonon band in Ge that prevents phonon polariton modes from transferring radiative heating from the hot to the code side.

To explore the radiative energy transfer mechanisms further, we plot the spectral radiative energy flux in Fig. 4 at a bias voltage of $0.5 \mathrm{~V}$ for three different gap distances. It can be seen that in the low-frequency region, shown in Fig. 4a, the net radiative heat transfer is negative since the chemical potential of photons is zero. The fluctuation is due to interferences in the $500 \mu \mathrm{m} \mathrm{Ge}$ substrate, in addition to the variation of the extinction coefficient as shown in Fig. 2b. The absolute value increases as the distance is reduced but not significantly. If $\mathrm{Ge}$ is replaced by $\mathrm{GaSb}$, then the phonon resonance will match and surface phonon polaritons will occur to enhance the reverse near-field radiative transfer [23]. The use of Ge can significantly suppress the reverse radiative heat transfer due to surface phonon polaritons especially for nanoscale gap distances [27]. For example, if $\mathrm{GaSb}$ were used as the ambient material, the maximum refrigeration rate would decrease by $15 \%$ at $d=10 \mu \mathrm{m}$ and $71 \%$ at $d=10 \mathrm{~nm}$. At high frequencies, the increased chemical potential of the biased diode gives a positive heat transfer or refrigeration effect, as shown in Fig. 4b. Compared with $d=10 \mu \mathrm{m}$, it is noted that the spectral energy flux at $d=600 \mathrm{~nm}$ stays almost the same in the high-frequency region. It is not surprising since the gap distance of $600 \mathrm{~nm}$ although much smaller than the thermal wavelength (around $10 \mu \mathrm{m}$ ) where phonon modes play a dominant role is comparable to the bandgap wavelength, meaning that $600 \mathrm{~nm}$ lies in the near-field regime for phonon modes but still in the far-field regime to some extent at high frequencies beyond the bandgap. The net result is a negative refrigeration rate as will be discussed later. However, if $d$ is reduced down to $100 \mathrm{~nm}$, the positive high-frequency energy exchange is increased 
considerably. Despite the fact that the negative low-frequency energy exchange is also enhanced somewhat, the refrigeration rate is significantly increased at the nanoscale as shown in Fig. 3.

Figure 5 gives the maximum refrigeration rate as a function of the gap distance, obtained by optimizing the applied voltage $V$ for each $d$ value. The maximum refrigeration rate stays positive when the gap distance exceeds about a micron or below half a micron, but becomes negative in the intermediate regime for $0.5 \mu \mathrm{m}<d<0.8 \mu \mathrm{m}$, as shown by the insert. When $d$ is greater than $5 \mu \mathrm{m}, Q_{\max }$ approaches a constant and tends to be independent of the gap distance as it further increases. This regime is called the far field since both interference and photon tunneling effects are negligible. With decreasing $d$, a wavy feature shows up due to the interference effects, which appear when $d$ is in the same magnitude as the wavelengths of interest, about $1.7 \mu \mathrm{m}$ that corresponds to the bandgap. Further decreasing $d$, a dip with the refrigeration rate as $-2.26 \mathrm{~W} / \mathrm{m}^{2}$ occurs at $600 \mathrm{~nm}$ as shown in the insert of Fig. 5. The competing radiative energy transfer in the low-frequency region and high-frequency region as presented in Fig. 4 can fully account for this phenomenon.

The temperature difference is set to $10 \mathrm{~K}$ in preceding calculations. One may wonder what the lowest achievable temperature through this electroluminescence refrigeration technique (assuming $T_{2}$ is fixed at $300 \mathrm{~K}$ near room temperature). The maximum refrigeration rate from the emitter versus the temperature difference $\Delta T=T_{2}-T_{1}$ is given in Fig. 6a, 6b, and $6 \mathrm{c}$ for $d=10 \mu \mathrm{m}, 0.1 \mu \mathrm{m}$, and $10 \mathrm{~nm}$, respectively. With increasing temperature difference or decreasing $T_{1}$, the difference 
between $\frac{1}{\mathrm{e}^{(\hbar \omega-q V) / k_{\mathrm{B}} T_{1}}-1}$ and $\frac{1}{\mathrm{e}^{\hbar \omega / k_{\mathrm{B}} T_{2}}-1}$ at a certain $V$ shrinks so that the positive energy exchange at high frequencies decreases while the negative energy exchange at low frequencies is enhanced, leading to a reduced cooling effect. When the net radiative energy exchange is below the input electric power, the refrigeration rate becomes negative and there will be no refrigeration but actually heating from the ambient to the $\mathrm{GaSb}$ diode. The lowest temperature one can achieve occurs when the refrigeration rate $Q_{\text {out }}$ becomes zero. For $d=10 \mu \mathrm{m}$, the maximum achievable temperature difference is $15.8 \mathrm{~K}$ (the lowest $T_{1}$ is $284.2 \mathrm{~K}$ ), beyond which the heat starts to flow in rather than being extracted from the diode emitter. Pushing the emitter and receiver into the nearfield with a gap distance of $100 \mathrm{~nm}$, the maximum temperature difference is increased to $24.6 \mathrm{~K}$ or the lowest achievable temperature $T_{1}=275.4 \mathrm{~K}$. Further reducing the gap distance to $10 \mathrm{~nm}$, the maximum $\Delta T$ reaches $29.4 \mathrm{~K}$ and the lowest $T_{1}$ becomes $270.6 \mathrm{~K}$. The greatly increased refrigeration rate at $100 \mathrm{~nm}$ and $10 \mathrm{~nm}$ as compared with the farfield scenario means that the positive high-frequency energy flux overwhelms the negative energy flow at low frequencies, as explained previously with Fig. 4. As regards the minimum allowed temperature, it should be noted that the positive energy flux at high frequencies can be optimized by varying the bias voltage, though more electric power will be needed as the temperature difference increases. However, the magnitude of the negative energy flux at low temperatures increases as $T_{1}$ is reduced. When $T_{1}$ is sufficiently low, the net cooling rate becomes zero. At nanoscale spacing, the lowest $T_{1}$ when refrigeration can still occur is pushed to much smaller values. It is also worth to note that for $\Delta T$ exceeding $10 \mathrm{~K}$, the refrigeration effect may show up only in the near- 
field regime. For example, for $T_{1}=280 \mathrm{~K}$, the net cooling rate is negative in the far field, and becomes positive only when the gap distance is shorter than $166 \mathrm{~nm}$. Hence, nearfield photon tunneling effects can also make the otherwise heating effect into refrigeration.

In previous analyses, the considered nonidealities include intrinsic nonradiative Auger recombination and contributions of sub-bandgap electromagnetic waves (lowfrequency thermal radiation). For practically biased semiconductors, defects are unavoidable and nontrinsic nonradiatiave recombinations like Shockely-Read-Hall recombination may exist. The refrigeration rate given in Eq. (8) considering nonintrinsic nonradiative recombinations $I_{\text {ni }}$ becomes

$$
Q_{\text {out }}=Q_{12}-\left(I_{\text {rad }}+I_{\text {Auger }}+I_{\text {ni }}\right) V
$$

The maximum tolerance percentage of nonintrinsic recombinations can be defined by setting $Q_{\text {out }}=0$ in Eq. (10) so that

$$
\eta_{\text {toler }}=\frac{I_{\mathrm{ni}}}{I_{\text {rad }}+I_{\text {Auger }}+I_{\text {ni }}}=1-\frac{I_{\text {rad }}+I_{\text {Auger }}}{Q_{12}} V
$$

Taking $T_{1}=290 \mathrm{~K}$, the maximum tolerance of nonintrinsic recombinations increases from $22.6 \%$ to $31.6 \%$ when the gap distance is reduced from $10 \mu \mathrm{m}$ to $10 \mathrm{~nm}$. Therefore, working in the near-field regime can relieve the stringent requirement of semiconductor's quality to some extent. It is worth to note that the tolerance of nonintrinsic recombinations is related to the coefficient of performance given in Eq. (9) without considering $I_{\text {ni }}$ by

$$
\frac{1}{\eta_{\mathrm{toler}}}=1+\frac{1}{\beta_{\mathrm{COP}}}
$$


In other words, the COP is enhanced from 0.29 in the far-field scenario to 0.46 when $d=$ $10 \mathrm{~nm}$. This demonstrates that photon tunneling or coupling of evanescent waves can help to increase not only the refrigeration rate, but also the COP and the tolerance of crystal quality.

\section{Conclusions}

The electroluminescent refrigeration is investigated when the spacing between the cold and hot regions are separated at the nanoscale considering a practical design. A multilayer model is developed by employing the fluctuation-dissipation theory and the dyadic Green's function method to consider the nonuniform distribution of the chemical potential of photons and nanoscale radiative transfer by both propagating and evanescent waves above and below the bandgap frequency. Pushing the cold forward biased diode and a hot object into the nanoscale regime, the refrigeration rate can be enhanced by two to three orders of magnitude. Moreover, the allowable temperature difference is broadened from $15.8 \mathrm{~K}$ to $24.6 \mathrm{~K}$. The unachievable refrigeration effect in the far field may become possible by reducing the gap distance down to the near-field regime. In addition, the stringent requirement of crystal quality to achieve a refrigeration effect can be relieved to certain extent. This work helps to deepen the understanding of

electroluminescent refrigeration at the nanoscale and opens a pathway to enhance the refrigeration rate, broaden the operation temperature range, and relieve the requirement of high-quality semiconductors. 


\section{Acknowledgements}

This work was supported by the Department of Energy, Office of Science, Basic Energy Sciences (DE-FG02-06ER46343). 


\section{References}

[1] M. Weinstein, J. Opt. Soc. Am. 50 (1960) 597-602.

[2] G. Dousmanis, C. Mueller, H. Nelson, K. Petzinger, Phys. Rev. 133 (1964) A316A318.

[3] S.-T. Yen, K.-C. Lee, J. Appl. Phys. 107 (2010) 054513.

[4] P. Berdahl, J. Appl. Phys. 58 (1985) 1369-1374.

[5] O. Heikkilä, J. Oksanen, J. Tulkki, J. Appl. Phys. 107 (2010) 033105.

[6] D. J. Gray Jr, P. Santhanam, R. J. Ram, Appl. Phys. Lett. 103 (2013) 123503.

[7] K.-C. Lee, S.-T. Yen, J. Appl. Phys. 111 (2012) 014511.

[8] A. Mal'shukov, K. Chao, Phys. Rev. Lett. 86 (2001) 5570-5573.

[9] P. Han, K.-J. Jin, Y. Zhou, X. Wang, Z. Ma, S.-F. Ren, A. Mal'shukov, K.-A. Chao, J. Appl. Phys. 99 (2006) 074504.

[10] S.-Q. Yu, J.-B. Wang, D. Ding, S. Johnson, D. Vasileska, Y.-H. Zhang, Solid-State Electron. 51 (2007) 1387-1390.

[11] J. Oksanen, J. Tulkki, J. .Appl. Phys. 107 (2010) 093106.

[12] P. Santhanam, D. J. Gray Jr, R. J. Ram, Phys. Rev. Lett. 108 (2012) 097403.

[13] S. M. Rytov, Y. A. Kravtsov, V. I. Tatarskii, Principles of Statistical Radiophysics, Springer, New York (1989).

[14] Z. M. Zhang, Nano/Microscale Heat Transfer, McGraw-Hill, New York (2007).

[15] Y. Xuan, Phot. Nano. Fund. Appl. 12 (2014) 93-113.

[16] K. Joulain, J. P. Mulet, F. Marquier, R. Carminati, J. J. Greffet, Surf. Sci. Rep. 57 (2005) 59-112.

[17] S. Shen, Annu. Rev. Heat Transfer 16 (2013) 327-343.

[18] X. L. Liu, L. P. Wang, Z. M. Zhang, Nanosc. Microsc. Thermophys. Eng. 19 (2015) 98-126.

[19] A. Kittel, W. Muller-Hirsch, J. Parisi, S. A. Biehs, D. Reddig, M. Holthaus, Phys. Rev. Lett. 95 (2005) 224301. 
[20] E. Rousseau, A. Siria, G. Jourdan, S. Volz, F. Comin, J. Chevrier, J. J. Greffet, Nat. Photon. 3 (2009) 514-517.

[21] S. Shen, A. Narayanaswamy, G. Chen, Nano Lett. 9 (2009) 2909-2913.

[22] R. St-Gelais, B. Guha, L. Zhu, S. Fan, M. Lipson, Nano Lett. 14 (2014) 6971-6975.

[23] K. Chen, P. Santhanam, S. Sandhu, L. Zhu, S. Fan, Phys. Rev. B 91 (2015) 134301.

[24] E. D. Palik, Handbook of Optical Constants of Solids, Academic Press, San Diego (1998).

[25] R. Ferrini, M. Patrini, S. Franchi, J. Appl. Phys. 84 (1998) 4517-4524.

[26] M. Wakaki, T. Shibuya, K. Kudo, Physical Properties and Data of Optical Materials, CRC Press, Boca Raton (2007).

[27] K. Chen, P. Santhanam, S. Fan, Appl. Phys. Lett. 107 (2015) 091106.

[28] Z. Hu, A. Perera, Y. Paltiel, A. Raizman, A. Sher, J. Appl. Phys. 98 (2005) 023511.

[29] P. Wurfel, J. Phys. C: Solid State Phys. 15 (1982) 3967-3985.

[30] C. H. Henry, R. F. Kazarinov, Rev. Mod. Phys. 68 (1996) 801-853.

[31] J. E. Sipe, J. Opt. Soc. Am. B 4 (1987) 481-489.

[32] K. Park, S. Basu, W. P. King, Z. M. Zhang, J. Quant. Spectrosc. Radiat. Transfer 109 (2008) 305-316.

[33] M. Francoeur, M. P. Mengüç, R. Vaillon, J. Quant. Spectrosc. Radiat. Transfer 110 (2009) 2002-2018.

[34] G. Stollwerck, O. V. Sulima, A. W. Bett, IEEE Trans. Electron Dev. 47 (2000) 448457.

[35] X. L. Liu, R. Z. Zhang, Z. M. Zhang, ACS Photon. 1 (2014) 785-789.

[36] S. A. Biehs, M. Tschikin, P. Ben-Abdallah, Phys. Rev. Lett. 109 (2012) 104301.

[37] Y. Guo, C. L. Cortes, S. Molesky, Z. Jacob, Appl. Phys. Lett. 101 (2012) 131106.

[38] X. L. Liu, Z. M. Zhang, ACS Photon. 2 (2015) 1320-1326. 


\section{Vitae of authors:}

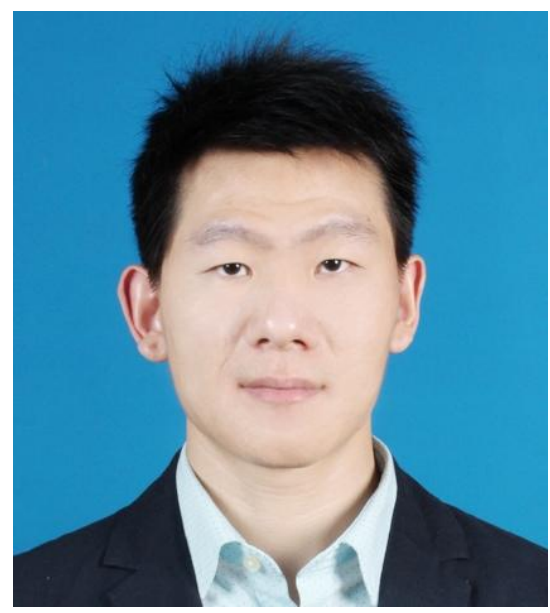

Xianglei Liu received his B.S. degree from Xi'an Jiaotong University and Ph.D. degree from Georgia Institute of Technology under the guidance of Professor Zhuomin Zhang. He now works in the School of Energy and Power Engineering of Nanjing University of Aeronautics and Astronautics. He has authored and co-authored over 20 peer-refereed journal papers. His research mainly focuses on radiation energy transfer, solar-thermal energy harvesting, Casimir interactions, and metamaterials.

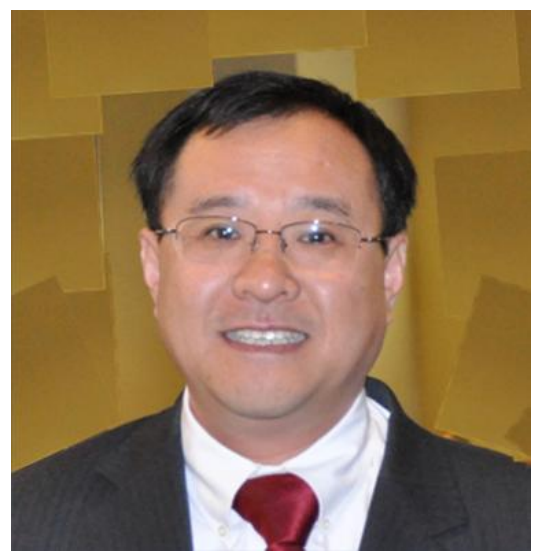

Zhuomin Zhang received his B.S. and M.S. degrees from the University of Science and Technology of China and Ph.D. degree from the Massachusetts Institute of Technology. $\mathrm{He}$ is a professor of mechanical engineering at Georgia Institute of Technology. Professor Zhang has published over 160 peer-reviewed journals and a book on Nano/Microscale Heat Transfer (McGraw-Hill, 2007), which has been translated to Chinese. His research interests are in micro/nanoscale heat transfer especially thermal radiation for energy conversion and temperature measurement. He is a Fellow of the American Society of Mechanical Engineers, American Physical Society, and American Association for the Advancement of Science. 


\section{Figure Captions:}

FIG. 1 (a) Schematic of a forward-biased $p-i-n$ GaSb diode separated with a $\mathrm{Ag}$ substrate with Ge deposited on top with a gap distance of $d$. The temperature of the diode is $T_{1}$, and is assumed to be lower than that of the top object. In all calculations, $h_{\mathrm{p}}=h_{\mathrm{n}}=0.1 \mu \mathrm{m}, h_{\mathrm{i}}=1 \mu \mathrm{m}, h_{\mathrm{Ge}}=500 \mu \mathrm{m}, T_{2}=300 \mathrm{~K}$. Unless specified, $T_{1}=290 \mathrm{~K}$. (b) Band diagram to show the working principle of electroluminescent refrigeration. Electrons (filled circles) and holes (unfilled circles) are driven to the active regime, where they either radiatively recombine (illustrated by the horizontal solid line with arrow) to create both propagating waves and evanescent waves or nonradiatively recombine (illustrated by the horizontal dashed line with arrow) and the energy is lost to phonons.

FIG. 2 Optical constants of intrinsic GaSb, doped GaSb, and Ge: (a) Refraction index $n$; (b) Extinction coefficient $k$.

FIG. 3 The refrigeration rate for the biased diode as a function of applied voltage at different gaps: (a) $d=10 \mu \mathrm{m}$, (b) $d=100 \mathrm{~nm}$, and (c) $d=10 \mathrm{~nm}$. The maximum refrigeration rate exists at each distance with a bias voltage ranging from $0.49 \mathrm{~V}$ to $0.61 \mathrm{~V}$. Significant enhancement in the refrigeration rate is seen when operated with nanoscale gap spacing.

FIG. 4 Spectral radiative energy flux for a bias voltage of $0.5 \mathrm{~V}$ : (a) Low-frequency region, showing a negative flux due to radiative heat transfer from higher to lower temperatures; (b) High-frequency region, showing a positive flux due to the change of photon energy potential beyond the bandgap.

FIG. 5 The maximum refrigerate rate from the biased diode versus the gap distance.

FIG. 6 The maximum refrigeration rate as a function of the temperature difference at different distances: (a) $d=10 \mu \mathrm{m}$, (b) $d=100 \mathrm{~nm}$, and (c) $d=10 \mathrm{~nm}$. 


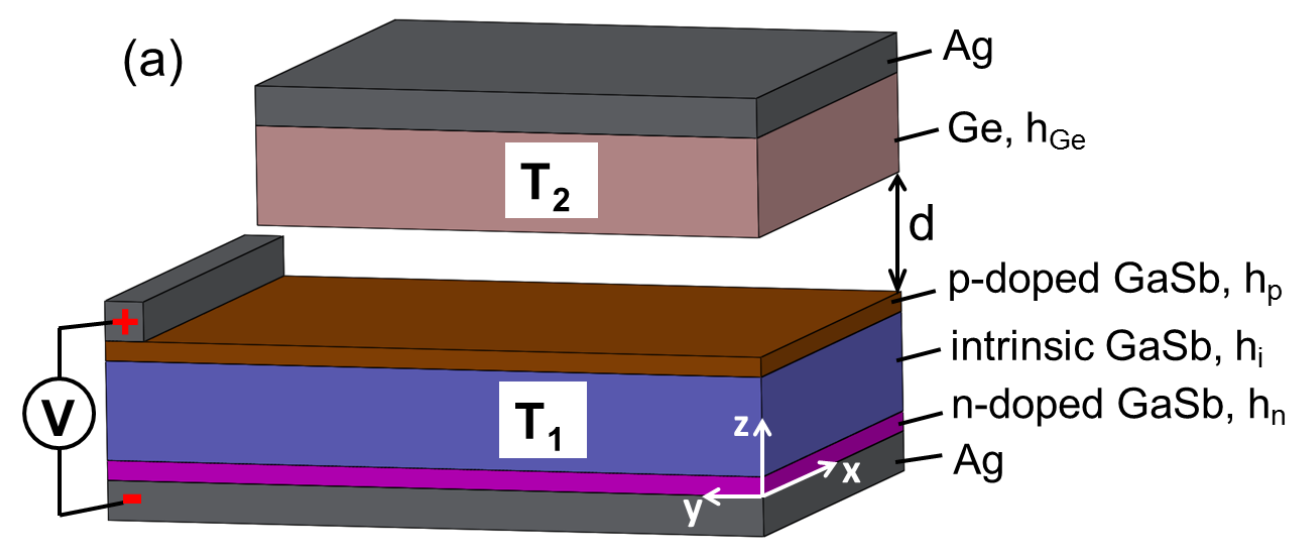

(b)

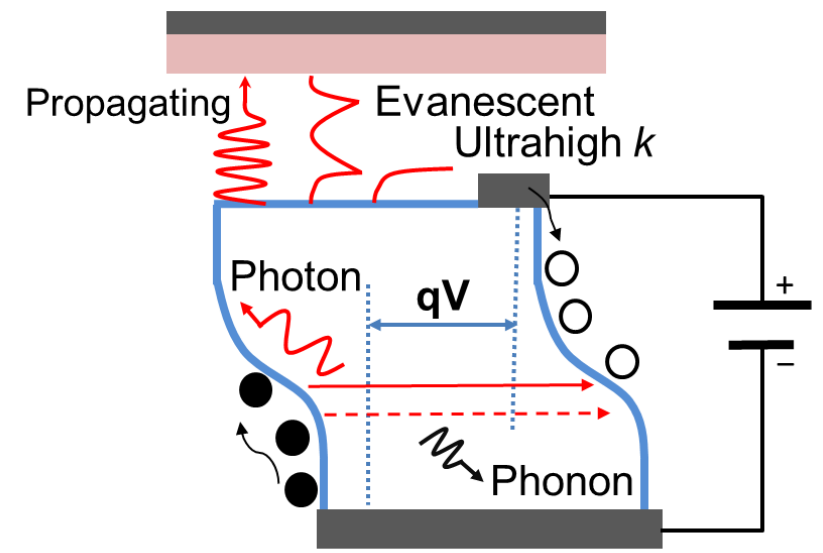

Liu and Zhang, Fig. 1 

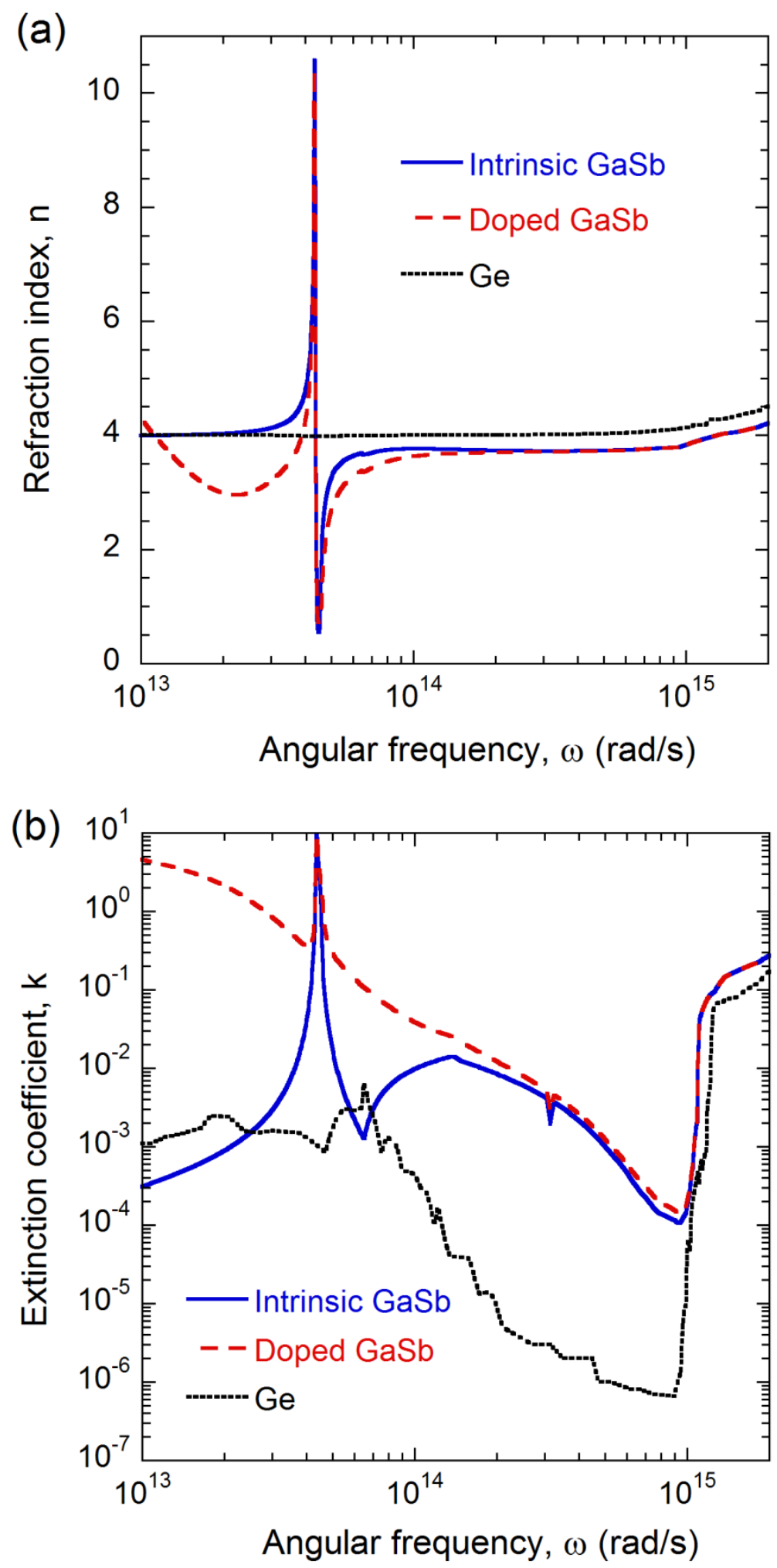

Liu and Zhang, Fig. 2 

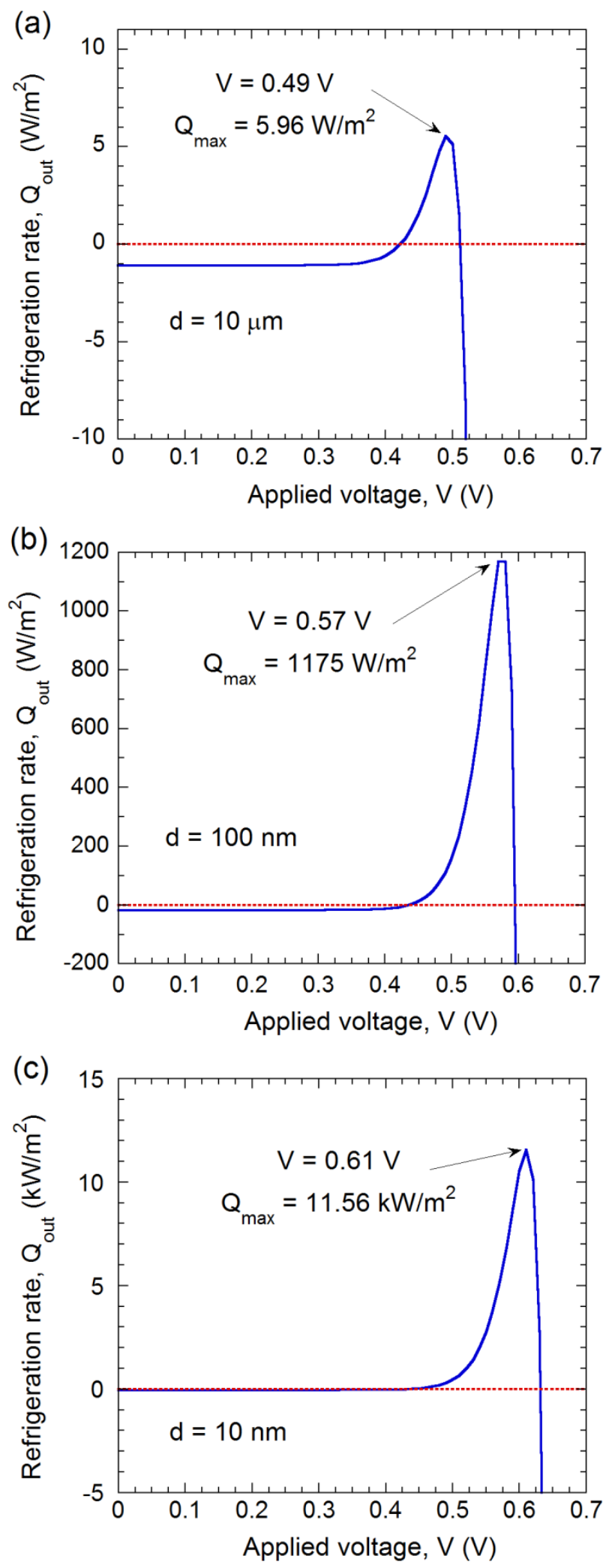

Liu and Zhang, Fig. 3 

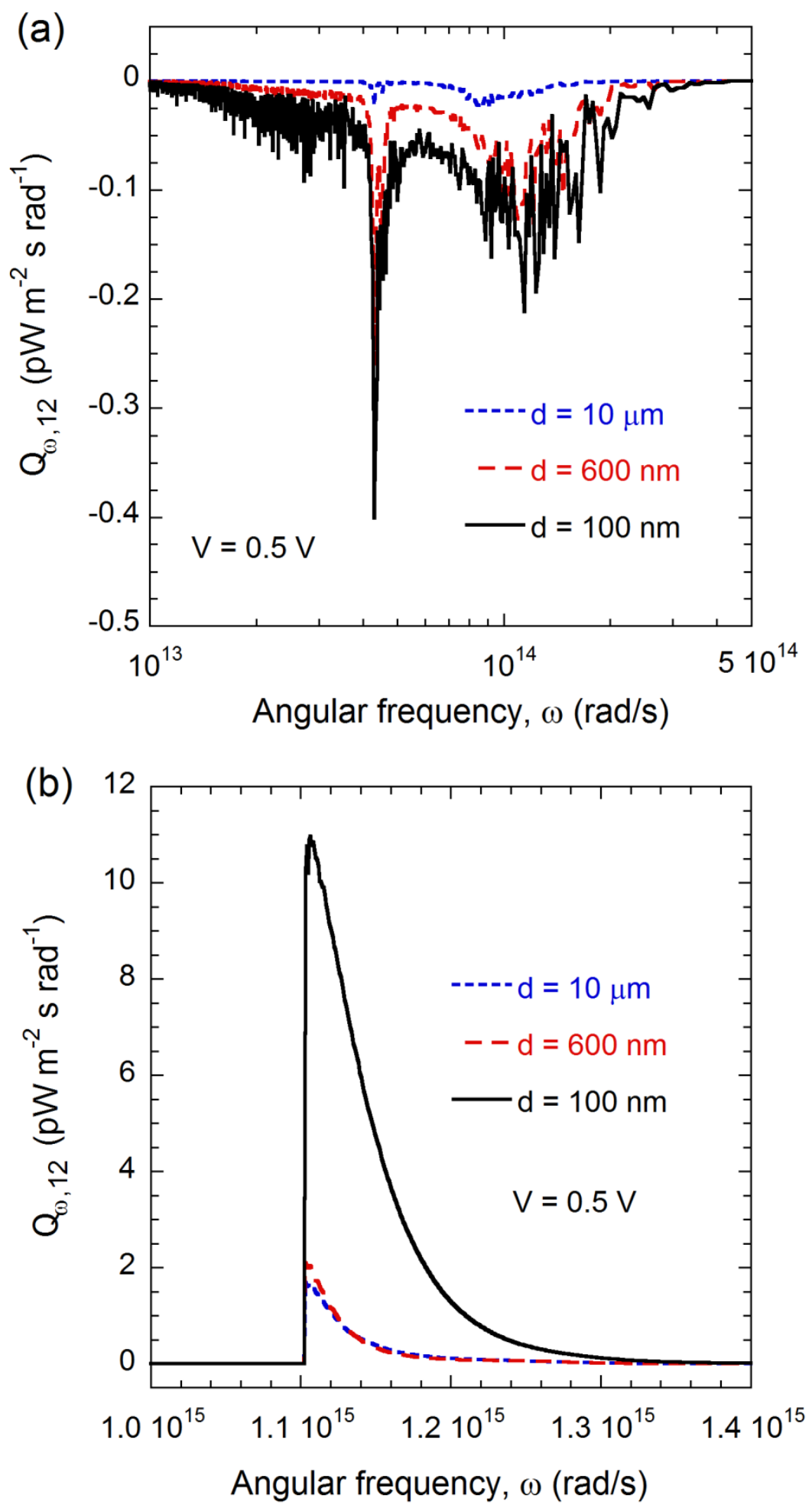

Liu and Zhang, Fig. 4 


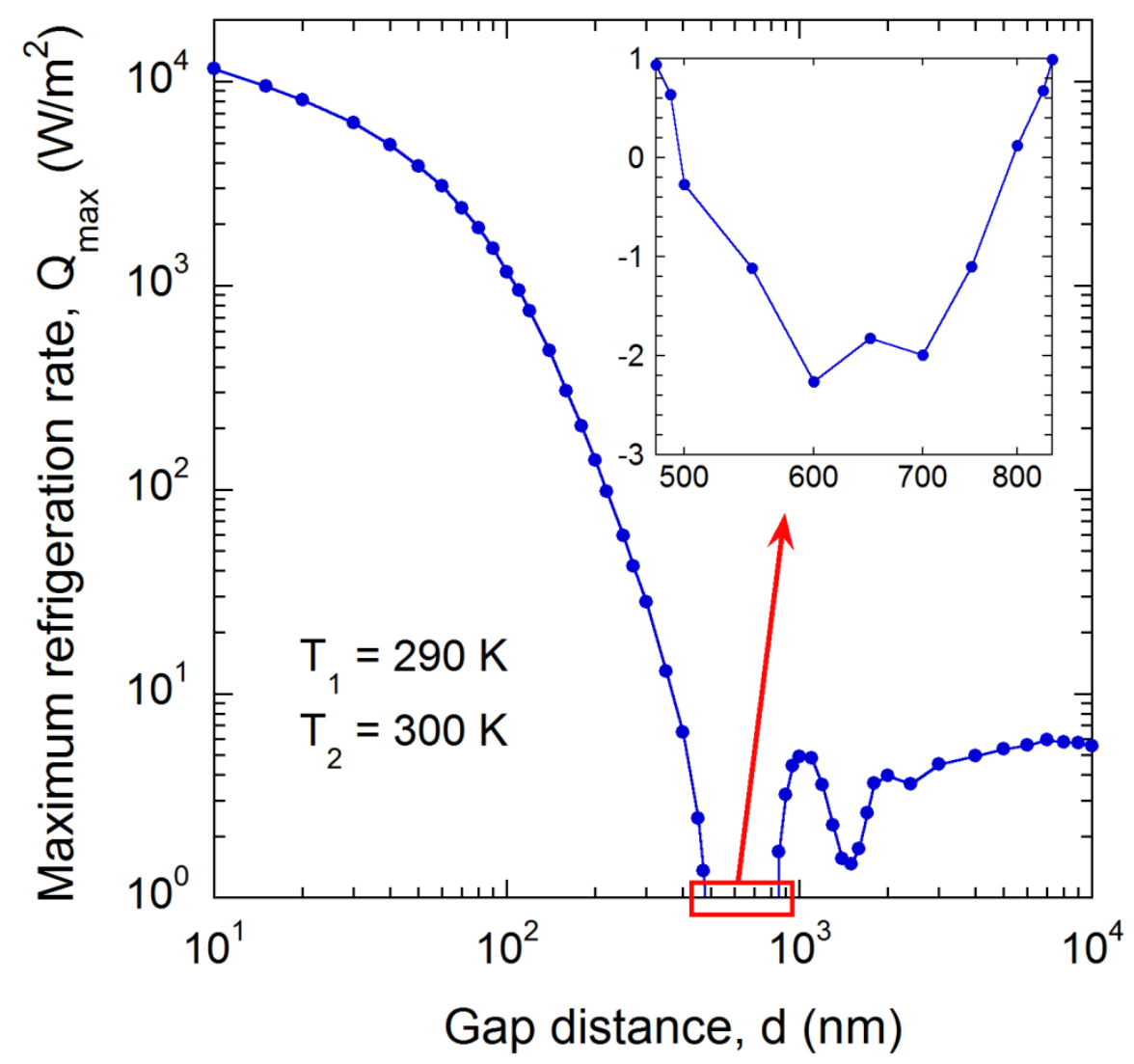

Liu and Zhang, Fig. 5 

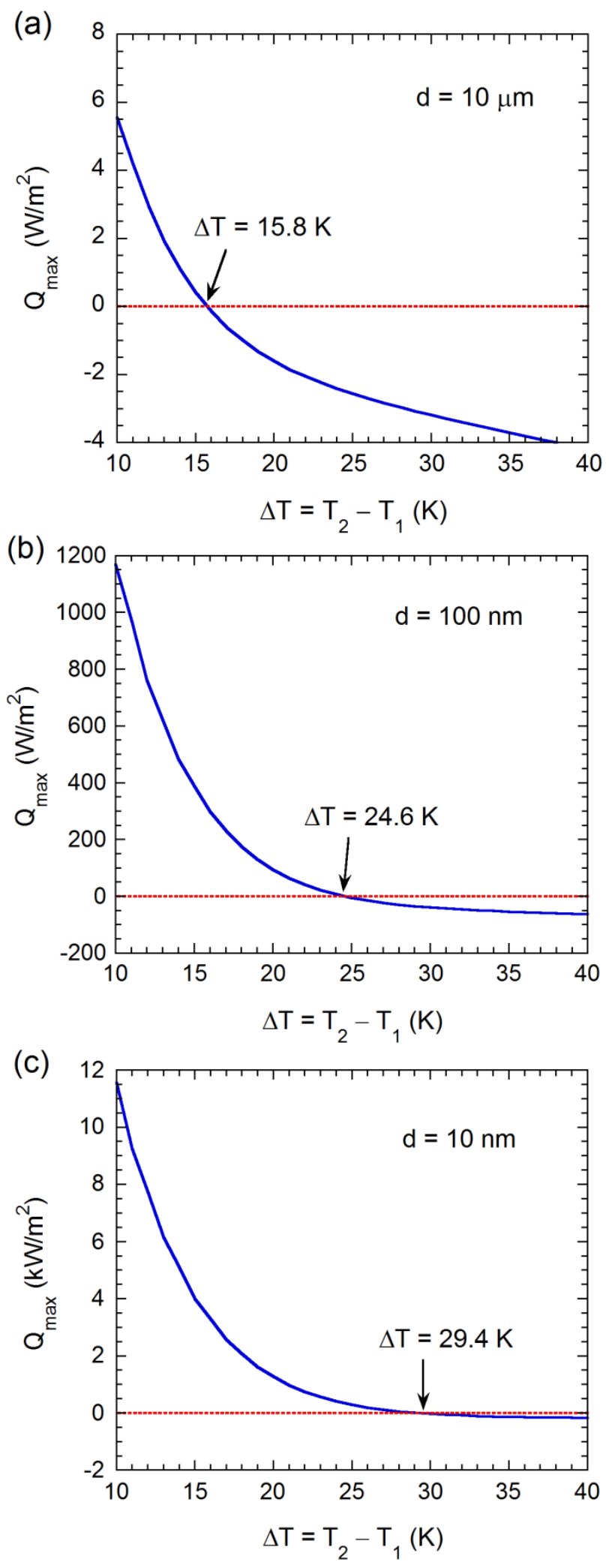

Liu and Zhang, Fig. 6 